\title{
Myocardial Revascularization in Critical Patients with Acute Myocardial Infarction and Cardiogenic Shock - a Perspective on New European Recommendations
}

\author{
Theodora Benedek
}

Clinic of Cardiology, University of Medicine, Pharmacy, Sciences and Technology, Tîrgu Mureș, Romania

Patients with multivessel disease and acute myocardial infarction (AMI) complicated with cardiogenic shock (CS) present one of the worst outcomes in the field of cardiac critical care, with mortality rates reported as high as 45$50 \%$ even in the presence of the most modern facilities for advanced cardiac support. ${ }^{1}$

The main recent directions of development in the field of emergency care for critical patients with CS and AMI are represented by: the change introduced by the new European recommendations regarding the revascularization of culprit lesions only in patients with AMI and CS, the implementation of regional networks for reducing time delays from symptom onset to revascularization, and the use of modern equipment for advanced cardiac support. ${ }^{1-3}$

The first major change in the therapeutic strategy for CS-AMI patients is related to the recent change in the European recommendation for primary revascularization in patients with multivessel disease and CS complicating AMI. Several years ago, the SHOCK (Should We Emergently Revascularize Occluded Coronaries for Cardiogenic Shock) trial demonstrated that emergency revascularization can significantly improve survival in patients with CS complicating AMI. ${ }^{2}$ However, in the presence of multivessel disease, there were inconclusive data to support the need for complete revascularization of all lesions in emergency conditions. ${ }^{3}$ Until recently, while major international guidelines recommended against urgent revascularization of non-culprit lesions in hemodynamically stable patients with STEMI, European guidelines recommended that revascularization of non-culprit arteries should be considered in patients with CS (indication class IIa). ${ }^{4}$

The recent guidelines on myocardial revascularization published by the European Society of Cardiology clearly advise against complete revascularization in patients with CS, recommending revascularization of the culprit lesion only (the lesion that caused infarction) and staged procedures for the non-culprit lesions. ${ }^{5}$ This change in recommendations is mainly caused by the recently published results of the CULPRIT-SHOCK trial, which demonstrated that in patients with multivessel disease, AMI, and CS, a strategy consisting in primary percutaneous coronary intervention (PCI) of the culprit lesion only is associated with a significant risk reduction in 30-day all-cause mortality ( $43.3 \%$ vs. $51.6 \%, \mathrm{HR}=0.84, \mathrm{p}=0.03$ ) and with a significantly lower incidence of the composite endpoint consisting in all-cause mortality or severe renal failure, compared to a strategy consisting in complete revascularization of all coronary lesions in emergency. ${ }^{6}$ Therefore, the current guidelines published this year strongly recommend culprit lesion-only primary PCI in patients with CS complicating AMI.

The second major direction for development in cardiac critical care for CS patients is represented by the efforts to implement appropriate logistics for reducing time de- 
lays from symptom onset to urgent revascularization. The recently published FITT-STEMI (Feed-back Intervention and Treatment Times in ST-Elevation Myocardial Infarction) trial demonstrated that every 10-minute delay from symptom onset to revascularization is associated with a $3.3 \%$ increase in mortality in patients with CS and with a $1.3 \%$ increase in mortality in patients with out-of-hospital cardiac arrest (OHCA), emphasizing the impact of an appropriate network and logistics in STEMI patients with CS or OHCA. ${ }^{7}$

The third direction for development addresses the critical issue represented by mechanical complications that can significantly increase mortality even in patients undergoing successful PCI. New devices for mechanical circulatory support, applied for a short term in refractory CS, may improve survival in these cases. Such devices include the intra-aortic balloon pump (IABP), veno-arterial extracorporeal membrane oxygenation (ECMO), and left ventricular assist devices (LVAD).

IABP can be inserted in the aorta during an interventional procedure, being able to decrease ventricular workload and to increase cardiac output. Despite the initial enthusiasm, large clinical trials failed to demonstrate any substantial benefit in reducing 30-day or longer-term mortality. Currently, the routine use of IABP in patients with AMI complicated with CS is not recommended.

ECMO devices are able to increase coronary, cerebral, and peripheral perfusion, acting as an extracorporeal life support device after being inserted percutaneously. Initial data shows promising results associated with the use of ECMO, which has been proved to be associated with a 33\% higher survival at 30 days compared with IABP in several studies. $^{8}$

Left ventricular assist devices are mainly dedicated to percutaneous implantation using a transaortic (Impella) or transseptal (TandemnHeart) route. The results obtained after using LVAD in patients with CS and AMI demonstrated similar mortality rates with IABP, failing to demonstrate any superiority in terms of MACE rates at 30 days in parallel with a significant increase in the rate of limb ischemia after implantation. ${ }^{9}$ Therefore, the use of percutaneous LVAD does not support the use of this device in patients with CS and AMI.

In conclusion, many efforts have been invested in recent years to decrease mortality in patients with AMI and
CS. These efforts are now reflected in a significant change in the guideline-based recommendations to treat patients in these critical conditions, the most important change being represented by the shift from complete revascularization to culprit-only revascularization recommendation in patients with CS and AMI.

\section{CONFLICT OF INTEREST}

Nothing to declare.

\section{REFERENCES}

1. Van Herck JL, Claeys MJ, De Paep R, Van Herck PL, Vrints CJ, Jorens PG. Management of cardiogenic shock complicating acute myocardial infarction. Eur Heart J Acute Cardiovasc Care. 2015;4:278-297. doi: 10.1177/2048872614568294.

2. HochmanJS, SleeperLA, WebbJG, etal.Early revascularizationin acute myocardial infarction complicated by cardiogenic shock. SHOCK Investigators. Should We Emergently Revascularize Occluded Coronaries for Cardiogenic Shock. N Engl J Med. 1999;341:625-634. doi: 10.1056/NEJM199908263410901.

3. Engstrom $\mathrm{T}$, Kelbaek $\mathrm{H}$, Helqvist $\mathrm{S}$, et al. Complete revascularisation versus treatment of the culprit lesion only in patients with ST-segment elevation myocardial infarction and multivessel disease (DANAMI-3-PRIMULTI): an openlabel, randomised controlled trial. Lancet. 2015;386:665-671.

4. Ibanez B, James S, Agewall S, et al. 2017 ESC Guidelines for the management of acute myocardial infarction in patients presenting with ST-segment elevation. Eur Heart J. 2018;39:119-177. doi: 10.1093/eurheartj/ehx393.

5. Sousa-Uva M, Neumann FJ, Ahlsson A, et al. 2018 ESC/EACTS Guidelines on myocardial revascularization. Eur J Cardiothorac Surg. 2019;55:4-90. doi: 10.1093/ejcts/ezy289.

6. Thiele H, Akin I, Sandri M, et al. PCI strategies in patients with acute myocardial infarction and cardiogenic shock. N Engl J Med. 2017;377:2419-2432. doi: 10.1056/NEJMoa1710261.

7. Scholz KH, Maier SKG, Maier LS, et al. Impact of treatment delay on mortality in ST-segment elevation myocardial infarction (STEMI) patients presenting with and without haemodynamic instability: Results from the German prospective, multicentre FITT-STEMI trial. Eur Heart J. 2018;39:1065-1074. doi: 10.1093/eurheartj/ehy004.

8. Ouweneel DM, Schotborgh JV, Limpens J, et al. Extracorporeal life support during cardiac arrest and cardiogenic shock: a systematic review and meta-analysis. Intensive Care Med. 2016;42:1922-1934. doi: 10.1007/s00134-016-4536-8.

9. Thiele H, Jobs A, Ouweneel DM, et al. Percutaneous shortterm active mechanical support devices in cardiogenic shock: A systematic review and collaborative meta-analysis of randomized trials. Eur Heart J. 2017;38:3523-3531. doi: 10.1093/eurheartj/ehx363. 